\title{
Cardiovascular risk factors among people with severe mental illness in Italy: \\ A cross-sectional comparative study.
}

\author{
Massimo Clerici ${ }^{1}$, Francesco Bartoli ${ }^{1}$, Daniele Carretta ${ }^{1}$, Cristina Crocamo ${ }^{1}$, Paul \\ Bebbington $^{2}$, Giuseppe Carrà ${ }^{2}$.
}

\section{Regular article resubmitted for publication to General Hospital Psychiatry}

Word count: Abstract 177; Text 3007; Tables: 2

${ }^{1}$ Department of Surgery and Interdisciplinary Medicine, University of Milano Bicocca, Monza. Italy.

${ }^{2}$ Division of Psychiatry, Faculty of Brain Sciences, University College London.

London, UK

\section{Corresponding author:}

Daniele Carretta, MD, Department of Surgery and Interdisciplinary Medicine, University of Milano-Bicocca, Via Cadore 48, 20900 Monza (MB), Italy.

E-mail: d.carretta1@ campus.unimib.it

Tel.: +39039233 3982 Fax: +390392332277 


\begin{abstract}
Objective: This study aimed to explore the prevalence of CVDs risk factors in people with severe mental illness (SMI), estimating its weight on related risks in Southern Europe, an area with distinct dietary traditions. People with SMI may be more likely than general population to have high prevalence of risk factors for cardiovascular diseases (CVDs), due to several reasons, including poor health behaviours and psychopharmacological medications.
\end{abstract}

Method: A cross-sectional study has been conducted, comparing inpatients with and without SMI. Univariate and multivariate analyses and logistic regression models have been carried out, exploring the effect of SMI on the prevalence of CVDs risk factors. Results: Although obesity and hypertriglyceridemia were more prevalent among people with SMI, controlling for relevant confounders in multivariate analyses we could not detect any significant difference.

Conclusions: People with SMI may not necessarily have higher CVDs risk, as compared with general population. Mediterranean healthy dietary habits, universal health coverage, families providing strong support, all are possible explanations for our findings. Further research is needed, exploring different mechanisms, with more detailed biological and genetics measures.

\title{
Keywords
}

Cardiovascular Diseases, Mediterranean diet, Mental Disorders/epidemiology; Comorbidity; Prevalence; Risk factors. 


\section{Background}

Over the past years, evidence has been accumulated showing that frequency of cardiovascular diseases (CVDs) risk factors -including hypertension, smoking, poor diet, obesity, lack of exercise, diabetes mellitus, and dyslipidemia- are often higher among people suffering from psychiatric disorders than in general population [e.g., 1-5]. In particular, people with severe mental illness (SMI) show a threefold increase in risk of developing diabetes mellitus as compared to general population [6,7]. Smoking prevalence can be up to four times higher among people with SMI than in general population [8]. Several studies report higher prevalence also of hypertension, dyslipidemia and metabolic syndrome among people with SMI, though differences do not always reach statistical significance [6,7]. Furthermore, in people with SMI as compared with general population, morbidity related to CVDs is up to three times higher [9], and this issue - along with associated mortality [10] - is cause for concern because of reduced life expectancy [11].

Several hypotheses have been proposed in order to explain the additional load in terms of CVDs risk factors in people with SMI [12]. SMI itself may genetically bring increased CVDs risk factors [13]. Also, unhealthy lifestyle and poor compliance to medications prescribed for physical illnesses [14] are likely to be involved. However, antipsychotics and other psychotropic medications by themselves play a major role, in hyperglycemia, increased serum cholesterol and weight gain [15]. Finally both clinicians and health services may pose barriers to accessing appropriate care, with people with SMI receiving physical treatment poorer than general population [12], despite lifestyle modification interventions and preventive programs maybe effective also for this special population [16]. 
Each of described etiological possibilities may be illuminated by studying them in geographical contexts with particular attributes. The population of countries in Southern Europe usually show reduced CVDs-related mortality rates, possibly because of generally healthy Mediterranean dietary habits, though other risk factors like smoking are more prevalent than in the U.S. [17]. This region thus offers opportunities for studying the prevalence of CVDs risk factors in relation to family and personal history, psychotropic medication and adverse health behaviors in people with SMI. If these are important causes of CVDs risk factors, the relative odds in people with SMI should be increased in the context of healthy eating habits at the population level. Understanding variations in prevalence of CVDs risk factors in people with SMI is important for several major reasons. First, geographical variation in CVDs risk factors in people with SMI is important in appreciating the impact of local social circumstances on prevalence, and whether variations are just as apparent as in the general population [18]. Secondly, good prevalence data are crucial to planning basic primary prevention and routine diagnostic screening for CVDs risk factors in this vulnerable group. Finally, in etiological terms, the relative roles of antipsychotic medication and of adverse health behaviors in relation to CVDs risk factors can be better studied in a context with traditionally healthy dietary habits. Unfortunately, earlier investigations in Southern Europe have been influenced by methodological issues. Samples have often been based on clinical convenience [19], and control groups have been unsuitable for a number of reasons, lacking relevant healthy comparison groups [20], using comparison samples from different populations [21] or based on large existing administrative databases [22]. This study aims to explore the prevalence of CVDs risk factors in a representative cohort of people with SMI in Italy. We hypothesized that after adjusting for relevant 
clinical and social variables, even in this healthy dietary context people with SMI would have CVDs risk factors prevalence rates significantly higher than those without mental disorders.

\section{Methods}

\section{Setting and study design}

We carried out a cross-sectional comparative study among people with and without SMI, recruited from inpatients admitted to San Gerardo University Hospital, whose 1,105 beds -with a comprehensive range of medical and surgical inpatient servicesinclude a 20 beds ward for acute mental health care, serving a catchment area of 319,000 inhabitants. The study took place in Monza-Brianza (MB), a mixed urban and rural province in Northern Italy, which comprises relatively wealthy districts, as compared with the national average based on 2011 annual Census data [23]. The research project complied with the principles of the Declaration of Helsinki regarding medical research in humans [24]. The local research ethics committee approved the study (code "Non solo mente").

\section{Sampling and procedures}

SMI was defined according to a modified version of the US Substance Abuse and Mental Health Service Administration definition [25], by requiring at least one 12month DSM-IV/SCID diagnosis other than a substance use disorder (i.e. Psychotic Disorders, Bipolar Disorder and Major Depressive Disorder with Psychotic Features), along with a Global Assessment of Functioning (GAF) score of less than 60 during the worst month of the past year [26,27]. All patients consecutively admitted between March 2011 and February 2012 to the mental health inpatient ward and with a SMI diagnosis, were approached for their informed and signed consent to the study. If they 
agreed, they were interviewed on the day before their planned discharge in order to check their diagnosis. Fully trained consultant psychiatrists administered the 12-month patient version of the SCID-I (Structured Clinical Interview for DSM-IV [28]) to identify SMI diagnoses within this group. Patients' functioning was assessed clinically and using medical records. In the same period, all inpatients from the maxillofacial surgery unit - consecutively admitted for planned (not emergency) cosmetic surgical treatment for minor or mild car accidents- were invited to participate in order to build an eligible list for the comparison group. We chose this clinical population as unlikely to suffer more from chronic physical disorders than the general population. To be eligible for recruitment, people from the index and the comparison groups had to be at least 18 years old and living in a private residence (rather than in an institutional setting). People from the comparison group were excluded if they had received a formal psychiatric diagnosis, had ever taken an antipsychotic, a mood stabilizer or an antidepressant medication, or were on the caseload of any mental health service nationwide. CVDs risk factors were defined according to cut-off values of the third report of the National Cholesterol Education Program Expert Panel on Detection, Evaluation, and Treatment of High Blood Cholesterol in Adults [29]:

- $\quad$ obesity (Body Mass Index $-\mathrm{BMI} \geq 30 \mathrm{~kg} / \mathrm{m} 2$ );

- hypertriglyceridemia (fasting triglycerides concentration $\geq 200 \mathrm{mg} / \mathrm{dl}$ );

- hypertension (systolic/diastolic blood pressure $\geq 140 / 90 \mathrm{mmHg}$ and/or on antihypertensive medication);

- $\quad$ low density lipoprotein (LDL) cholesterol $\geq 160 \mathrm{mg} / \mathrm{dl}$ );

- hyperglycemia (fasting blood glucose level $\geq 126 \mathrm{mg} / \mathrm{dl}$ or on insulin or hypoglycemic medication). 
Finally, we estimated total cholesterol/HDL cholesterol ratios, and the whole sample was dichotomized around the relevant median value, giving a high and a low ratio category [30].

\section{Data collection}

Patients' data were obtained for index and control subgroups from both clinical records and interviews. We collected general socio-demographic information for each participant. We systematically recorded clinical data including current smoker's status [31], family and personal history of CVDs (i.e. myocardial infarction, acute heart failure, acute coronary syndrome, and stroke), diagnoses of hypertension, dyslipidemia and diabetes mellitus. For SMI inpatients we gathered information about current medication, and amounts of antipsychotic agents prescribed in the previous six months were converted in average chlorpromazine (CPZ) equivalent doses, according to the best available sources [32-35], though the validity of this measure is not entirely clear [e.g., 36]. Mood stabilizer and antidepressant medications were also collected. As part of the routine admission procedure, height and weight (on electronic scales, with the minimal necessary clothing) were measured, and BMI was calculated. If feasible, we retrieved waist circumference with a non-elastic measuring tape around the abdomen at the approximate midpoint between the lower margin of the last palpable rib and the top of the iliac crest [37]. We measured blood pressure at the beginning and end of the interview using an automated sphygmomanometer, with the patient in a sitting position after at least 5 minutes resting, and the mean value was determined. Blood samples were drawn around $8.00 \mathrm{am}$, and the patients had fasted at least for the previous 10 hours. Plasma levels of glucose, triglycerides and total, LDL, HDL cholesterol were analyzed. All serum analyses were performed using standard methods on a Cobas 8000 , 
module c701 (Roche Diagnostics, Basel, Switzerland) at the Department of Clinical Biochemistry of San Gerardo University Hospital, Monza (MB), Italy.

\section{Statistical analysis}

Statistical analyses were completed using Stata version 10.0 SE. We performed comparative analyses between two samples, each one drawn with a simple random sampling technique from the enrolled populations. Further details on sampling and randomization procedures have been described elsewhere [38].

We carried out univariate analyses to identify patients' attributes characteristic of SMI. The normality of continuous data was checked with Shapiro-Wilk's test. If the variances of the two subgroups examined were not homogeneous, we used the t-test for unequal variances and the Welch's approximation of the degrees of freedom. Student's t-test was performed for normally distributed continuous data. Chi-square and Fisher's exact tests were used for categorical variables. As regards the effect of SMI on the prevalence of CVDs risk factors we followed a structured approach. First we estimated unadjusted ORs (95\% CIs) for each CVD risk factor. Then, we added chlorpromazine equivalent dose as possible explanation for the increased rates of CVD risk factors in people with SMI, using Sobel-Goodman tests for mediation as framed in the sgmediation command in Stata. We used mediation analyses to test whether the relationship between SMI (independent variable) and each CVD risk factor (dependent variables) is direct or whether the mediator of chlorpromazine equivalent dose accounts for the relationship between them. If the association between SMI and each CVD risk factor is not reduced by a significant amount (partial mediation) by the mediator chlorpromazine equivalent dose, all that can be inferred is a direct effect of SMI on CVD risk factors. This would justify carrying out logistic regressions of the effect of SMI on the prevalence of 
relevant CVDs risk factors, controlling for chlorpromazine equivalent dose as well as for age and gender. Finally we carried out logistic regressions of the effect of SMI on the prevalence of relevant CVDs risk factors, controlling for all sociodemographic and clinical covariates that were significantly $(\mathrm{p}<0.1)$ related in the univariate analyses.

\section{Results}

\section{Characteristics of participants}

In total, 298 SMI patients and 264 comparison group patients were initially identified, of whom 208 and 196 were found to be eligible in the SMI and the comparison group respectively, all belonging to the white Italian ethnic group. None of the users screened for eligibility refused to participate in the study. Valid data were available for 119 patients with SMI (Psychotic Disorders=82, Bipolar Disorders=31; Major Depressive Disorder with Psychotic Features=6) and 119 without SMI. The socio-demographic and clinical characteristics associated with SMI are summarized in Table 1. People with SMI were significantly more often males and current smokers but less married or cohabiting and fewer participants were employed. They also had mean levels of BMI, waist circumference, fasting triglycerides and total/HDL cholesterol ratio significantly higher than controls. They also showed significantly lower mean systolic blood pressure. Other variables did not reach statistical significance. In total, 115 out of 119 patients $(97 \%)$ in the SMI group were taking antipsychotics, 110 of which were atypical (26 Risperidone, 18 Quetiapine, 32 Olanzapine, 12 Clozapine, 10 Aripiprazole, 6 Paliperidone, 6 Ziprasidone). The median chlorpromazine dose was $242 \mathrm{mg}$ (interquartile range (IQR) 150-483 mg).

\section{TABLE 1 about here}

\section{Multivariate analysis}


In relation to the main CVDs risk factors, as defined by NCEP ATP III, only prevalence of obesity and hypertriglyceridemia were higher in those with SMI than in those without (Table 2). We then tested our specific hypotheses relating to potential mediator role of antipsychotic medication dose of the relationship between SMI and each CVDs risk factor. However, the proportion of the effect of SMI on each CVDs risk factor that was mediated by antipsychotic medication dose was minuscule, and not statistically significant: virtually all the effect was, therefore, direct. Thus, in our data, there is no evidence that antipsychotic medication dose can explain the excess of CVDs risk factors in people with SMI. Thus it was justifiable to add in the logistic regression models, age, gender, and chlorpromazine equivalent as covariates. The magnitude of our results changed, with hypertriglyceridemia remaining significantly higher, as well as total cholesterol/HDL cholesterol ratio, in people with SMI. Finally, ORs were adjusted for all sociodemographic and clinical covariates that were significantly related in the univariate analyses to each risk factor (these differed slightly in relation to the different factors but with appreciable overlap). However, none of CVDs risk factors maintained conventional levels of significance, with hypertriglyceridemia and total /HDL cholesterol ratio, showing just a marginal trend.

TABLE 2 about here

\section{Discussion}

\section{Main findings}

We analyzed an epidemiologically representative random sample of inpatients with SMI. An appropriate comparison group was established and chosen. DSM-IV research diagnoses for SMI were based on formal diagnostic interviews. We were thus able to 
study SMI in relation to differences in rates of CVDs risk factors in a region with distinct dietary traditions but also poor healthy behaviors such as smoking. The absence of mediation by antipsychotic dose may suggest that, at the population level at least, such drugs do not contribute much to the excess, or perhaps that much of the medication effect is already apparent at low dosage. People with SMI in our sample were significantly more often current smokers and also had mean levels of BMI, waist circumference, fasting triglycerides and total/HDL cholesterol ratio significantly higher than controls. They also showed significantly lower mean systolic blood pressure. Though in the univariate analyses our data showed that hypertriglyceridemia and total cholesterol/HDL cholesterol ratio were significantly higher in people with SMI, none of CVDs risk factors maintained conventional levels of significance when controlling for all sociodemographic and clinical covariates that were significantly related in the univariate analyses to each risk factor. However, adjusting for relevant covariates, we could not find any association, though hypertriglyceridemia and total/HDL cholesterol ratio showed a non-significant trend in people with SMI.

Our findings do not support previous research from other Western countries. SMI has been found to be associated with obesity, smoking, diabetes mellitus, hypertension and metabolic syndrome [10,14,39], and generally the prevalence of CVDs risk factors among people with SMI is high [40]. However, obesity and hypertriglyceridemia do not seem inherent to SMI, since it can be effectively managed by specific interventions, including dietary education and physical activity [41], but also other types of interventions such as health education, smoking cessation, and changes in health care organization [42]. Indeed also a relatively recent meta-analysis found that only diabetes mellitus was strongly associated with SMI, while hypertension and dyslipidemia were 
not, even if studies about the latter two factors were too heterogeneous or inadequate to draw firm conclusions [7].

There are several possible alternative explanations for our findings. First National Health System universal health coverage, available to all, regardless of wealth, may make possible effective prevention also for people with SMI in terms of CVDs risk factors [43]. However, comparable data, from well established NHS (U.K. and Norway), still show CVDs risk factors rates significantly higher in people with SMI than in general practice comparison groups [30,44]. Secondly, in Italy, as in other Mediterranean countries, family provides strong support for people with SMI [45] perhaps positively influencing dietary habits. Finally, Mediterranean healthy dietary habits could be important protective factors, reducing CVDs risk factors, such as hypertriglyceridemia and insulin resistance $[38,46]$. However, findings on CVDs risk factors rates in people with SMI from other Mediterranean countries are not homogeneous, and our data seem comparable with some [20] but not all $[19,21,22,47]$ studies. Along with methodological issues, it may well be that Mediterranean healthy dietary habits in different Southern Europe countries are influenced by others factors such as area-level socio-economic position and wealth [48].

\section{Limitations}

The procedure for identifying SMI was based on the use of a standardized instrument, the SCID though the reliability of ratings was not formally established during the study. We have addressed selection bias at the stage of design for the index and the comparison groups (random sampling) and the role of possible confounders at the stage of analysis for the whole sample (adjusting for a selected set of variables). While attempts were made to guarantee comparable recruitment procedures, acute-phase 
treatment might have exacerbated the metabolic disturbances, and in general our results may not be generalizable beyond hospitalized populations, that could possibly experience poor social or familial support, which could thereby influence their diet. Given the cross-sectional design of our study, no reverse causality is allowed, though it is unlikely that CVD risk factors may cause SMI. Prospective time series data, with comprehensive assessment of lifetime exposure to antipsychotics, are needed to answer the question of causation, and we could consider for our analyses only psychopharmacological medications prescribed in the last six-months before admission, not to mention unknown compliance and other comorbid conditions $[49,50]$. However, though differences emerged between groups with and without SMI, we could study the differential confounding effects, and their mechanisms, of antipsychotic medication, of health behaviors such as smoking, and an indirect indicator of inherited risk (family history of CVDs, though it could just as likely be due to shared environment as genetics). The setting, the manner of delivery of interventions and the exclusion criteria are relatively similar to those found elsewhere, allowing reasonable generalizability to similar populations in Western countries. Nevertheless, as CVDs risk factors were assessed in treated populations, findings might not be the same in people out of contact with services. Also, we did not collect information on dietary habits of our sample, although poor diet partly accounts for the higher incidence of metabolic abnormalities among people with schizophrenia [1].

\section{Conclusions}

In a relatively wealthy area, with traditionally healthy dietary habits, CVDs risk factors among people with SMI may not be more prevalent than in the general population. 
Further research in Southern Europe countries should consider different mechanisms, with more detailed biological and genetics measures, in order to explore other potential protective factors, specific of people with SMI and shared with general population. 


\section{References}

[1] Di Pasquale S, Pariante CM, Dazzan P et al. The dietary pattern of patients with schizophrenia: a systematic review. J Psychiatr Res 2013;47:197-207.

[2] Mitchell AJ, Vancampfort D, Sweers K et al. Prevalence of metabolic syndrome and metabolic abnormalities in schizophrenia and related disorders--a systematic review and meta-analysis. Schizophr Bull 2013;39:306-18.

[3] Vancampfort D, Vansteelandt K, Correll CU et al. Metabolic syndrome and metabolic abnormalities in bipolar disorder: a meta-analysis of prevalence rates and moderators. Am J Psychiatry. 2013;170:265-74.

[4] Bartoli F, Carrà G, Crocamo C, Carretta D, Clerici M. Metabolic syndrome in people suffering from posttraumatic stress disorder: a systematic review and metaanalysis. Metab Syndr Relat Disord 2013;11:301-308.

[5] Bartoli F, Carrà G, Crocamo C, Carretta D, Clerici M. Bipolar disorder, schizophrenia, and metabolic syndrome. Am J Psychiatry. 2013;170:927-8.

[6] De Hert M, Dekker JM, Wood D et al. Cardiovascular disease and diabetes in people with severe mental illness position statement from the European Psychiatric Association (EPA), supported by the European Association for the Study of Diabetes (EASD) and the European Society of Cardiology (ESC). Eur Psychiatry 2009;24:412-424.

[7] Osborn DP, Wright CA, Levy G et al. Relative risk of diabetes, dyslipidaemia, hypertension and the metabolic syndrome in people with severe mental illnesses: systematic review and metaanalysis. BMC Psychiatry 2008;8:84.

[8] Filia SL, Baker AL, Gurvich CT, Richmond R, Kulkarni J. The perceived risks and benefits of quitting in smokers diagnosed with severe mental illness participating in a smoking cessation intervention: gender differences and comparison to smokers without mental illness. Drug Alcohol Rev 2014;33:78-85.

[9] Van Winkel R, Van Os J, Celic I et al. Psychiatric diagnosis as an independent risk factor for metabolic disturbances: results from a comprehensive, naturalistic screening program. J Clin Psychiatry 2008;69:1319-1327.

[10] Allison DB, Newcomer JW, Dunn AL et al. Obesity among those with mental disorders - A National Institute of Mental Health meeting report. Am J Prev Med 2009;36:341-350. 
[11] Lawrence D, Hancock KJ, Kisely S. The gap in life expectancy from preventable physical illness in psychiatric patients in Western Australia: retrospective analysis of population based registers. BMJ 2013; 21;346:f2539. doi: 10.1136/bmj.f2539.

[12] Leucht S, Burkard T, Henderson J, Maj M, Sartorius N. Physical illness and schizophrenia : a review of the literature. Acta Psychiatr Scand 2007;116:317-333.

[13] Gough SCL, O’Donovan MC. Clustering of metabolic comorbidity in schizophrenia: a genetic contribution? J Psychopharmacol 2005;19 suppl:47-55.

[14] Hennekens CH, Hennekens AR, Hollar D, Casey DE. Schizophrenia and increased risks of cardiovascular disease. Am Hearth J 2005;150:1115-1121.

[15] Falissard B, Mauri M, Shaw K et al. The METEOR study: frequency of metabolic disorders in patients with schizophrenia. Focus on first and second generation and level of risk of antipsychotic drugs. Int Clin Psychopharmacol 2011;26:291-302.

[16] Fernández-San-Martín MI, Martín-López LM, Masa-Font R et al. The effectiveness of lifestyle interventions to reduce cardiovascular risk in patients with severe mental disorders: meta-analysis of intervention studies. Community Ment Health J2014;50:81-95.

[17] Kokkinos P, Panagiotakos DB, Polychronopoulos E. Dietary influences on blood pressure: the effect of the Mediterranean diet on the prevalence of hypertension. $\mathrm{J}$ Clin Hypertens (Greenwich) 2005;7:165-170.

[18] Chimonas T, Karagiannis A, Athyros VG et al. Blood pressure levels constitute the most important determinant of the metabolic syndrome in a Mediterranean population: a discrimination analysis. Metab Syndr Relat Disord 2010;8:523-529.

[19] Salvi V, Albert U, Chiarle A et al. Metabolic syndrome in Italian patients with bipolar disorder. Gen Hosp Psychiatry 2008;30:318-323.

[20] Fusar-Poli P, De Marco L, Cavallin F, Bertorello A, Nicolasi M, Politi P. Lifestyles and cardiovascular risk in individuals with functional psychoses. Perspect Psychiatr Care 2009;45:87-99.

[21] Yazici MK, Anil Yağcioğlu AE, Ertuğrul A et al. The prevalence and clinical correlates of metabolic syndrome in patients with schizophrenia: findings from a cohort in Turkey. Eur Arch Psychiatry Clin Neurosci 2011;261:69-78.

[22] Cicero AF, Forghieri M, Cipressi F, Arletti R. Cardiovascular risk factors in severely ill psychiatric patients. Epidemiol Prev 2003;27:40-43. 
[23] Istituto Nazionale di Statistica [ISTAT] (2011). Annuario statistico italiano 2011. Retrieved from http://www3.istat.it/dati/catalogo/20111216_00/asi2011.zip

[24] World Medical Association. WMA Declaration of Helsinki: ethical principles for medical research involving human subjects. Ferney-Voltaire, France: WMA 2008. Retrieved from http://www.wma.net/en/30publications/10policies/b3/index.html

[25] Alcohol, Drug Abuse, and Mental Health Administration (ADAMHA - 1992). Reorganization Act, Pub. L. No. 102-321.

[26] Endicott J, Spitzer RL, Fleiss JL, Cohen J. The Global Assessment Scale: a procedure for measuring the overall severity of psychiatric disturbance. Arch Gen Psychiatry 1976;33:766-771.

[27] Carrà G, Sciarini P, Segagni-Lusignani G, Clerici M et al. Do they actually work across borders? Evaluation of two measures of psychological distress as screening instruments in a non Anglo-Saxon country. Eur Psychiatry 2011;26:122-127.

[28] Spitzer RL, Williams JB, Gibbon M, First MB. The Structured Clinical Interview for DSM-III-R (SCID). I: History, rationale, and description. Arch Gen Psychiatry 1992;49:624-629.

[29] National Cholesterol Education Program. Third report of the National Cholesterol Education Program (NCEP) Expert Panel on detection, evaluation and treatment of high blood cholesterol in adults (Adult Treatment Panel III) final report. Circulation 2002;106:3143.

[30] Osborn DPJ, Nazareth I, King MB. Risk for coronary heart disease in people with severe mental illness: cross-sectional comparative study in primary care. Br J Psychiatry 2006;188:271-277.

[31] Centers for Disease Control and Prevention. State-specific secondhand smoke exposure and current cigarette smoking among adults-United States, 2008. MMWR Morb Mortal Wkly Rep. 2009;58:1232-1235.

[32] Atkins M, Burgess A, Bottomley C, Riccio M. Chlorpromazine equivalents: a consensus of opinion for both clinical and research applications. Psychiatr Bull R Coll Psychiatr 1997;21:224-226.

[33] Lehman AF, Steinwachs DM. Translating research into practice: the Schizophrenia Patient Outcomes Research Team (PORT) treatment recommendations. Schizophr Bull 1998;24:1-10. 
[34] Woods SW. Chlorpromazine equivalent doses for the newer atypical antipsychotics. J Clin Psychiatry 2003;64:663-667.

[35] Kroken RA, Johnsen E, Ruud T, Wentzel-Larsen T, Jørgensen HA. Treatment of schizophrenia with antipsychotics in Norwegian emergency wards, a cross-sectional national study. BMC Psychiatry 2009;9:24.

[36] Andreasen NC, Pressler M, Nopoulos P, Miller D, Ho BC. Antipsychotic dose equivalents and dose-years: a standardized method for comparing exposure to different drugs. Biol Psychiatry 2010;67:255-262.

[37] World Health Organization [WHO] (2011). Waist circumference and waist-hip ratio: report of a WHO expert consultation. Geneva, 8-11 December 2008. Geneva, Switzerland: WHO Retrieved from http://whqlibdoc.who.int/publications/2011/9789241501491_eng.pdf

[38] Carrà G, Bartoli F, Carretta D et al. The prevalence of metabolic syndrome in people with severe mental illness. Soc Psychiatry Psychiatr Epidemiol. 2014 doi: 10.1007/s00127-014-0835-y.

[39] Keenan TE, Yu A, Cooper LA et al. Racial patterns of cardiovascular disease risk factors in serious mental illness and the overall U.S. population. Schizophr Res 2013;150:211-216.

[40] Osborn DP, Baio G, Walters K et al. Inequalities in the provision of cardiovascular screening to people with severe mental illnesses in primary care. Cohort study in the United Kingdom THIN Primary Care Database 2000-2007. Schizophr Res 2011;129:104-110.

[41] Chacón F, Mora F, Gervas-Rios A, Gilaberte I. Efficacy of lifestyle interventions in physical health management of patients with severe mental illness. Ann Gen Psychiatry 2011;10:22.

[42] Van Hasselt FM, Krabbe PFM, van Ittersum DG, Postma MJ, Loonen AJM. Evaluating interventions to improve somatic health in severe mental illness: a systematic review. Acta Psychiatr Scand 2013;128:251-260.

[43] Apolone G, Lattuada L. Health coverage in Italy. J Ambul Care Manage 2003;26:378-382.

[44] Birkenaes AB, Opjordsmoen S, Brunborg C, Engh JA, Jonsdottir H, Ringen PA, Simonsen C, Vaskinn A, Birkeland KI, Friis S, Sundet K, Andreassen OA. The 
level of cardiovascular risk factors in bipolar disorder equals that of schizophrenia: a comparative study. J Clin Psychiatry 2007;68:917-923.

[45] Gonçalves-Pereira M, Xavier M, van Wijngaarden B et al. Impact of psychosis on Portuguese caregivers: a cross-cultural exploration of burden, distress, positive aspects and clinical-functional correlates. Soc Psychiatry Psychiatr Epidemiol 2013;48:325-335.

[46] Bonaccio M, Iacoviello I, de Gaetano G; Moli-Sani Investigators. The Mediterranean diet: The reasons for a success. Thromb Res 2012;129:401-404.

[47] Sicras-Mainar A, Blanca-Tamayo M, Rejas-Gutiérrez J, Navarro-Artieda R. Metabolic syndrome in outpatients receiving antipsychotic therapy in routine clinical practice: a cross-sectional assessment of a primary health care database. Eur Psychiatry 2008;23:100-108.

[48] Meer J, Miller DL, Rosen HS. Exploring the health-wealth nexus. J Health Econ. 200;22:713-730.

[49] Carrà G, Johnson S. Variations in rates of comorbid substance use in psychosis between mental health settings and geographical areas in the UK. A systematic review. Soc Psychiatry Psychiatr Epidemiol 2009;44:429-447.

[50] Carrà G, Johnson S, Bebbington $\mathrm{P}$ et al. The lifetime and past-year prevalence of dual diagnosis in people with schizophrenia across Europe: findings from the European Schizophrenia Cohort (EuroSC). Eur Arch Psychiatry Clin Neurosci 2012;262:607-616. 
Table 1. Socio-demographic and clinical characteristics associated with severe mental illness (SMI)

\begin{tabular}{|c|c|c|c|}
\hline Variable & $\begin{array}{c}\text { With SMI } \\
(n=119)\end{array}$ & $\begin{array}{c}\text { Without SMI } \\
(n=119)\end{array}$ & $\boldsymbol{P}$ \\
\hline Age: Mean (SD), yrs & $47.2(14.8)$ & $49.4(19.7)$ & $\mathrm{ns}^{\mathrm{a}}$ \\
\hline Gender: No. (\%) Male & $51(42.9)$ & $65(54.6)$ & $0.069 *$ \\
\hline Education: No. (\%) $\geq$ High school & $58(48.7)$ & $56(47.1)$ & $\mathrm{ns} *$ \\
\hline Married/cohabiting: No. (\%) & $28(23.5)$ & $64(54.7)$ & $<0.001 *$ \\
\hline Ordinary employed: No. (\%) & $58(48.7)$ & $104(87.4)$ & $<0.001 *$ \\
\hline BMI$^{1}:$ Mean (SD), kg/m2 & $25.5(6.6)$ & $24.2(4.3)$ & $0.075^{\mathrm{b}}$ \\
\hline Waist circumference: Mean(SD), cm & $95.2(17.1)$ & $91.0(14.8)$ & $0.052^{\dagger}$ \\
\hline 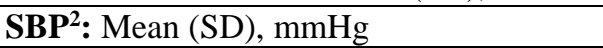 & $116.9(11.7)$ & $122.3(14.2)$ & $0.001^{\mathrm{c}}$ \\
\hline DBP $^{3}$ : Mean (SD), $\mathrm{mmHg}$ & $75.2(7.8)$ & $76.3(8.8)$ & $\mathrm{ns}^{\dagger}$ \\
\hline Fasting glucose: Mean (SD), mg/dl & $93.0(20.4)$ & $97.2(22.4)$ & $\mathrm{ns}^{\dagger}$ \\
\hline Triglycerides: Mean (SD), mg/dl & $121.9(91.5)$ & $97.9(52.9)$ & $0.014^{\mathrm{d}}$ \\
\hline Total cholesterol: Mean (SD), mg/dl & $183.3(46.8)$ & $179.7(40.4)$ & $\mathrm{ns}^{\dagger}$ \\
\hline LDL $^{4}:$ Mean (SD), mg/dl & $110.9(47.1)$ & $108.4(36.1)$ & $\mathrm{ns}^{\mathrm{e}}$ \\
\hline HDL $^{5}$ : Mean (SD), mg/dl & $48.4(14.8)$ & $51.5(14.9)$ & $\mathrm{ns}^{\dagger}$ \\
\hline Total cholesterol/HDL ratio: Mean (SD) & $4.1(1.6)$ & $3.7(1.4)$ & $0.079^{\dagger}$ \\
\hline Smoking: No (\%) & $77(64.7)$ & $63(52.9)$ & $0.065^{*}$ \\
\hline History of CVDs: (No. \%) & $11(9.2)$ & $14(11.8)$ & ns* \\
\hline Family history of CVDs: (No. \%) & $44(37.0)$ & $47(39.5)$ & $\mathrm{ns}^{*}$ \\
\hline
\end{tabular}

${ }^{1}$ Body mass index; ${ }^{2}$ Systolic blood pressure; ${ }^{3}$ Diastolic blood pressure; ${ }^{4}$ Low density lipoprotein; ${ }^{5} \mathrm{High}$ density lipoprotein. Student's $t$ test with unequal variances; Welch's degrees of freedom ${ }^{\mathrm{a}} 220.542$; 203.929 ; ${ }^{\mathrm{c}} 229.217$; ${ }^{\mathrm{d}} 190.333$; ${ }^{2} 222.836$

* Pearson's $\chi^{2}$ test with 1 d.f. 'Student's t test ( $\mathrm{df}=236$ for all) 
Table 2. Association between CVDs risk factors and SMI: univariate and multivariate analyses

\begin{tabular}{|c|c|c|c|}
\hline Variable & $\begin{array}{c}\text { Unadjusted OR } \\
(95 \% \mathrm{CI})\end{array}$ & $\begin{array}{l}\text { OR adjusted for } \\
\text { age, gender and } \\
\mathrm{CPZ}^{\dagger}(95 \% \mathrm{CI})\end{array}$ & $\begin{array}{l}\text { OR adjusted for age, } \\
\text { gender, CPZ and } \\
\text { variables significant at } \\
\text { the univariate level } \\
(95 \% \mathrm{CI})\end{array}$ \\
\hline $\begin{array}{l}\text { Obesity }^{1} \\
\quad \text { SMI } 21(17.6 \%) \\
\quad \text { Non-SMI } 10(8.4 \%) \\
\end{array}$ & $\begin{array}{c}\mathbf{2 . 3 4} * \\
(1.04-5.25) \\
\mathrm{P}=0.034 \\
\end{array}$ & $\begin{array}{c}\mathbf{2 . 2 2} \\
(0.89-5.52) \\
P=0.085 \\
\end{array}$ & $\begin{array}{c}\mathbf{1 . 6 9}^{1} \\
(0.62-4.58) \\
P=0.302 \\
\end{array}$ \\
\hline $\begin{array}{l}\text { Hypertension } \\
\text { SMI } 26(21.8 \%) \\
\text { Non-SMI } 39(32.7 \%)\end{array}$ & $\begin{array}{c}\mathbf{0 . 5 7} \\
(0.32-1.03) \\
\mathrm{P}=0.059\end{array}$ & $\begin{array}{c}\mathbf{0 . 5 8} \\
(0.26-1.29) \\
P=0.179\end{array}$ & $\begin{array}{c}\mathbf{0 . 5 8}^{2} \\
(0.24-1.41) \\
P=0.230\end{array}$ \\
\hline $\begin{array}{l}\text { Hyperglycaemia } \\
\text { SMI } 13(10.9 \%) \\
\text { Non-SMI } 16(13.9 \%)\end{array}$ & $\begin{array}{c}\mathbf{0 . 7 9} \\
(0.36-1.73) \\
P=0.553\end{array}$ & $\begin{array}{c}\mathbf{1 . 1 4} \\
(0.44-2.96) \\
P=0.784\end{array}$ & $\begin{array}{c}\mathbf{1 . 4 1}^{3} \\
(0.50-3.96) \\
P=0.514\end{array}$ \\
\hline $\begin{array}{c}\text { Hypertriglyceridemia } \\
\text { SMI } 18(15.1 \%) \\
\text { Non-SMI } 8(6.7 \%) \\
\end{array}$ & $\begin{array}{c}\mathbf{2 . 4 7} * \\
(1.03-5.93) \\
\mathrm{P}=0.04\end{array}$ & $\begin{array}{c}\mathbf{3 . 9 9} * \\
(1.39-11.48) \\
P=0.010 \\
\end{array}$ & $\begin{array}{c}\mathbf{3 . 1 1} 4 \\
(0.98-9.88) \\
P=0.055\end{array}$ \\
\hline $\begin{array}{l}\text { LDL Hypercholesterolemia } \\
\text { SMI } 14(11.7 \%) \\
\text { Non-SMI } 13(10.9 \%)\end{array}$ & $\begin{array}{c}\mathbf{1 . 0 9} \\
(0.49-2.43) \\
\mathrm{P}=0.838\end{array}$ & $\begin{array}{c}\mathbf{1 . 2 5} \\
(0.49-3.15) \\
P=0.639\end{array}$ & $-* *$ \\
\hline $\begin{array}{l}\text { Total /HDL cholesterol ratio } \\
\text { SMI } 66(55.5 \%) \\
\text { Non-SMI } 53(44.5 \%)\end{array}$ & $\begin{array}{c}\mathbf{1 . 5 5} \\
(0.93-2.60) \\
\mathrm{P}=0.093\end{array}$ & $\begin{array}{c}\mathbf{2 . 1 5} \\
(1.15-4.02) \\
P=0.016\end{array}$ & $\begin{array}{c}\mathbf{1 . 8 7}^{5} \\
(0.92-3.77) \\
P=0.081\end{array}$ \\
\hline
\end{tabular}

†equivalent dose of chlorpromazine for antipsychotic. *Statistically significant; **No variable was significantly associated with LDL hypercholesterolemia at the univariate analysis.

${ }^{1}$ Controlled for education, family history of cardiovascular disease, mood stabilizer medication; ${ }^{2}$ Controlled for family situation, education, current employment, history of cardiovascular disease, family history of cardiovascular disease; ${ }^{3}$ Controlled for family situation, history of cardiovascular disease, family history of cardiovascular disease; ${ }^{4}$ Controlled for smoking status, history of cardiovascular disease, mood stabilizer medications; ${ }^{5}$ Controlled for family situation, smoking status, antidepressant medications. 